\title{
Association between Brachial-Ankle pulse wave velocity and cardiac autonomic neuropathy in type 2 diabetes
}

Nan Wu${ }^{1}$, Xiaoling Cai ${ }^{2}$, Kuanping Ye ${ }^{1}$, Yintao $\mathrm{Li}^{1}$, Min He${ }^{1}$, Weiwei Zhao ${ }^{1}$ and Renming $\mathrm{Hu}^{{ }^{*}}$

\begin{abstract}
Background: Cardiac autonomic neuropathy (CAN) is a common complication of type 2 diabetes mellitus (T2DM). Brachial-ankle pulse wave velocity (baPWV) is known to be a good surrogate marker of vascular damages. The goal of this study was to investigate the relationship between BaPWV and CAN in T2DM.

Methods: A total of 148 patients who had no apparent history of cardiovascular condition were enrolled consecutively in this study. The correlation between increased baPWV and CAN was analyzed. CAN was evaluated by five standard cardiovascular reflex tests (CARTs) according to the Ewing's protocol: 1) heart rate variation during deep breathing, 2) heart rate response to standing, 3) Valsalva maneuver, 4) postural systolic blood pressure (BP) change, 5) Sustained handgrip test. CAN was defined as the presence of at least two abnormal tests.

Results: The mean age of patients was $59.8 \pm 7.8$ years. The mean duration of diabetes was 6.0(2.0-11.0) years. The mean baPWW was $1665.5(1482.0-1940.0) \mathrm{cm} / \mathrm{sec}$. Subjects with CAN were older and had high BMl, baPWV compared with those without CAN. The proportion of patients with diabetic peripheral neuropathy was higher in subjects with CAN. After adjusting for other confounding risk factors, baPWW (odds ratio $=8.496,95 \% \mathrm{Cl}: 1.216-59.348 ; \mathrm{P}=0.031$ ) remained as independent risk factors for CAN. The number of abnormal CARTs increased gradually with increasing baPWW (correlation coefficient $=0.255, p=0.002$ ).
\end{abstract}

Conclusion: Increased baPW was significantly correlated with CAN in patients with type 2 diabetes.

Keywords: Cardiac autonomic neuropathy, Brachial-ankle pulse wave velocity, Type 2 diabetes

\section{Introduction}

Cardiovascular autonomic neuropathy (CAN) is a serious complication of diabetes, which was associated with cardiovascular morbidity and all-cause mortality in people with Type 2 diabetes mellitus [1,2]. CAN was caused by damage to the autonomic nerve fibers that innervate the heart and blood vessels. It leads to dysfunctional heart-rate control and abnormal vascular dynamics, which will increase heart rate variability and decrease myocardial perfusion [3].

Pulse wave velocity (PWV) is known to be an indicator of arterial stiffness and a surrogate marker of vascular damages [4]. As brachial-ankle PWV (baPWV) is affected

\footnotetext{
* Correspondence: renminghu@hotmail.com

'The Institute of Endocrinology and Diabetology, Huashan Hospital, Shanghai Medical College, Fudan University, 12 Middle Wulumuqi Road, Shanghai, China

Full list of author information is available at the end of the article
}

by vasomotor reflexes, it can reflect the statements of the aorta and peripheral arteries. The baPWV has been thought to have a great association with micro-angiopathic conditions and diabetes complications [5]. Previous reports indicated that baPWV is used as a severity index for subclinical atherosclerosis [6], and has been recommended as test for the assessment of target organ damage in arterial hypertension [7]. Aso et al. showed that baPWV was directly related to the frequencies of albuminuria, autonomic neuropathy, peripheral neuropathy, and retinopathy [5]. Bagherzadeh et al. reported that there exists a significant relationship between heart rate variability (HRV) and baPWV in diabetic patients [8].

This study used a retrospective analysis to investigate CAN in type 2 diabetes patients and the association between baPWV and cardiovascular autonomic reflex tests (CARTs). 


\section{Method}

\section{Study subject}

We enrolled consecutive type 2 diabetes subjects (mean age. 59.8 years) who presented to outpatient department of endocrinology at Huashan Hospital between August 1, 2012 and February 30, 2013. Exclusion criteria included Type 1 diabetes characterized by a low C-peptide level $(<0.3 \mathrm{ng} / \mathrm{ml})$ and a history of diabetic ketoacidosis, other secondary causes of diabetes mellitus, malignancy, hepatic failure, acute metabolic complications, fatal arrhythmia, CVD such as acute coronary syndrome and previous myocardial infarction, cervical spondylosis, infectious polyneuritis, vasculitis, uremia, foot ulcers. Finally, 148 patients were enrolled in our study. Written informed consents were obtained from all participants and the study was approved by the local ethics committee of the Huashan Hospital.

\section{Data collection}

A standardized questionnaire was designed to collect clinical information regarding the duration of diabetes, alcohol consumption, cigarette smoking and other health related variables. Body weight and height were measured with the participant wearing light clothing without shoes. Body mass index (BMI) was defined as weight in kilogram divided by square height in meter. Waist circumference was measured at the narrowest point between the lower limit of the ribcage and the iliac crest. Hip circumference was measured around the widest portion of the buttocks, with the tape parallel to the floor. Blood pressure (BP) was measured in the supine position and in a resting state using mercury sphygmomanometer with an appropriate cuff on the left arm and the average of the last two measurements was used.

A 12-hour overnight fasting venous blood sample was collected for measurement of plasma glucose, HbA1c, creatinine, total cholesterol, low-density lipoprotein cholesterol (LDL-C), high-density lipoprotein cholesterol (HDL-C), triglycerides (TG) using standard protocol. First morning urine was collected once a day for 3 consecutive days. Albumin-to-creatinine radio was calculated by dividing urinary albumin by urinary creatinine.

\section{Cardiac autonomic function test}

Patients were instructed to refrain from alcohol, tobacco, caffeinated beverages and vigorous exercise during the 24 hours prior to the test. Before the test, the patients would fast at least 3 hours. Medications such as $\beta$ Blockers,antihistamines, antidepressants were withdrawn for 12 hours prior to the test. All the tests were performed by the same operator.

CAN was assessed by five standard cardiovascular reflex tests according to Ewing's protocol [9]. These included heart rate (HR) responses to deep breathing (beat to beat variation), lying to standing HR responses (30:15 ratio), Valsalva maneuver, blood pressure(BP) responses to standing and sustained handgrip test. The heart rate response to deep breathing, standing, and the Valsalva maneuver were assessed automatically from electrocardiography recordings using the Sunrui evaluation system (ECG-2203B, Sunrui Co., Ltd., Guangzhou, China). The severity of CAN was quantified by summing the points obtained from each of the five tests, where each test scored with $0,0.5$, or 1 points depending on whether it yielded normal, borderline, or abnormal values, respectively. CAN was defined as the presence of at least two abnormal tests or autonomic neuropathy points $\geq 2$ [10]. The CAN score was categorized as follows: CAN score 0 (total points 0 ), CAN score 1 (points 0.5 to 1.5 ), CAN score 2 (points 2 to 3 ), and CAN score 3 (points $\geq 3.5$ ). CAN was considered absent, early, definite, or severe if the CAN scores were $0,1,2$, or 3 , respectively.

\section{Measurements of baPWV}

VP-1000 Automatic Arteriosclerosis Measurement System (model BP-203 RPE-II, Colin Co, Ltd, Komaki, Japan) was used to measure BaPWV. The details of the measurement, validity, and reproducibility have been testified previously [11]. The device can record pulse waves by sensors in four cuffs, store data on the start point of each pulse wave in the right arm and both legs, record the time difference between transmission time to arm and transmission time to ankle as "transmission time", calculate the transmission distance from the right arm to each ankle according to body height, and automatically compute the baPWV values by transmission time and transmission distance. Patients were instructed to have a 5 min rest in a supine position in a room with air-condition $\left(24-26^{\circ} \mathrm{C}\right)$ before test. There was a significant positive correlation between left and right baPWV $(\mathrm{r}=0.953, \mathrm{P}<0.001)$. As a result, we used a mean of bilateral baPWV value during analysis.

\section{Evaluation of diabetic microvascular complications}

Diabetic retinopathy (DR) was defined based on digital non-mydriatic fundus photography protocol which was modified from procedures used in previous studies [12]. The patients were given $5 \mathrm{~min}$ in a darkened room to allow dark adaptation. A trained photographer took a single undilated non-mydriatic digital photograph centred on the fovea of each fundus using a Canon CR6-45NM camera, repeated once only if necessary. The digitally stored fundus photographs were stored as bitmap images and viewed in a darkened room on CRT screens at a resolution of $1024 \times 768$ pixels. The digitally stored fundus images were graded by two endocrinologists and a retinal specialist. Retinopathy was classified as either absent or present. 
The presence of diabetic nephropathy was defined as microalbuminuria (creatinine $30-300 \mathrm{mg} / \mathrm{g}$ ) or overt albuminuria (creatinine $>300 \mathrm{mg} / \mathrm{g}$ ). Urine albumin excretion was determined by measuring the urine albumin: creatinine ratio in spot urine samples [13].

Diabetic peripheral neuropathy (DPN) was assessed using Neuropathy Deficit Score (NDS) and Neuropathy Symptom Score (NSS). The diagnosis of DPN depends on both subjective symptoms and signs of neuropathy. We defined DPN as at least moderate signs with or

\section{Table 1 Clinical and biochemical characteristics of participants}

\begin{tabular}{|c|c|}
\hline Characteristic & $N=148$ \\
\hline cardiovascular autonomic neuropathy(\%) & $78(52.7)$ \\
\hline Age(years) & $59.8 \pm 7.8$ \\
\hline Male,n(\%) & $80(54.0)$ \\
\hline Current smoker, n (\%) & $43(29.0)$ \\
\hline Alcohol consumption, n (\%) & $37(25.0)$ \\
\hline Hypertension, n (\%) & $81(54.7)$ \\
\hline Use of $\beta$-Blockers & $34(22.9)$ \\
\hline Use of $A C E l / A R B$ & $67(45.3)$ \\
\hline Use of CCB & $48(32.4)$ \\
\hline Other antihypertensives & $14(9.5)$ \\
\hline Express as median(years) & $6.0(2.0-11.0)$ \\
\hline $\mathrm{BMI}\left(\mathrm{kg} / \mathrm{m}^{2}\right)$ & $24.4 \pm 3.4$ \\
\hline Waist-to-hip ratio & $0.94(0.90-0.99)$ \\
\hline systolic blood pressure(mmHg) & $134.2 \pm 18.1$ \\
\hline diastolic blood pressure $(\mathrm{mmHg})$ & $78.3 \pm 10.6$ \\
\hline $\mathrm{HbA} 1 \mathrm{c}(\%)$ & $8.0 \pm 1.9$ \\
\hline fasting glucose (mmol/l) & $7.1(5.8-9.2)$ \\
\hline Creatinine $(\mu \mathrm{mol} / \mathrm{L})$ & $62.0(53.0-78.0)$ \\
\hline $\mathrm{eGFR}\left(\mathrm{ml} / \mathrm{min} / 1.73 \mathrm{~m}^{2}\right)$ & $107.0 \pm 32.8$ \\
\hline total cholesterol(mmol/l) & $4.89 \pm 1.52$ \\
\hline Triglyceride(mmol/l) & $1.61(1.05-2.34)$ \\
\hline LDL cholesterol(mmol/l) & $2.84(2.29-3.60)$ \\
\hline HDL cholesterol(mmol/l) & $1.09(0.93-1.33)$ \\
\hline $\mathrm{ACR}(\mathrm{mg} / \mathrm{g})$ & $12.02(7.18-19.54)$ \\
\hline baPWV(cm/sec) & $1665.5(1482.0-1940.0)$ \\
\hline Diabetic retinopathy, n (\%) & 25(16.9) \\
\hline Diabetic peripheral neuropathy, n (\%) & $46(31.1)$ \\
\hline NSS score & $5.0(2.0-6.0)$ \\
\hline NDS score & $4.0(2.0-5.0)$ \\
\hline Diabetic nephropathy, n (\%) & $33(22.2)$ \\
\hline
\end{tabular}

BMI, body mass index; eGFR, modification of diet in renal disease study-glomerular filtration rate; $A C R$, albumin-to-creatinine ratio; $A C E I / A R B$, angiotensin converting enzyme inhibitors/Angiotensin II receptor blockers; CCB, Calcium channel blocker; NSS, neuropathy symptom score; NDS, neuropathy disability score. Data are means SD, median (25-75\%), or number (percent). without symptoms (NDS $\geq 6$ ), or mild signs with moderate symptoms (NDS $\geq 3$ and NSS $\geq 5$ ) [14].

\section{Statistical analyses}

Analyses were performed using IBM SPSS 19.0 for Windows (SPSS Inc., an IBM company, USA). Numerical variables with normal distribution are expressed as means $\pm \mathrm{SD}$, and reported as median (interquartile range) and log transformed to approximate normality before analysis otherwise. Pearson correlational analysis was used to examine the relationships between baPWV and other metabolic variables in Type 2 diabetes. The independent-samples $t$ test or chi-squared tests were used to compare the differences in clinical and biochemical characteristics between patients with and those without CAN. Multivariate logistic regression analyses were performed to estimate the contribution of baPWV to cardiac autonomic neuropathy using the odds ratio and 95\% CI. P values $<0.05$ were considered statistical significant.

\section{Results}

\section{General characteristics of participants}

The clinical and biochemical characteristics of the study population are shown in Table 1. A total number of 148 Chinese (80 men and 68 women) were included. Mean age, BMI and the duration of diabetes were $59.8 \pm 7.8$ years, $24.4 \pm 3.4 \mathrm{~kg} / \mathrm{m} 2$, and $7.2 \pm 5.8$ years, respectively. Mean baPWV was $1665.5(1482.0-1940.0) \mathrm{cm} / \mathrm{sec}$. Prevalence rates

Table 2 Correlation between baPWV and other clinical variables

\begin{tabular}{lll}
\hline \multicolumn{1}{c}{ Variable } & Correlation coefficients & P \\
\hline Age & 0.404 & 0.001 \\
Duration of diabetes* $^{*}$ & 0.141 & 0.095 \\
BMI & 0.124 & 0.134 \\
Waist-to-hip ratio* & 0.081 & 0.327 \\
systolic blood pressure & 0.654 & 0.001 \\
diastolic blood pressure(mmHg) & 0.327 & 0.001 \\
HbA1c & -0.010 & 0.900 \\
fasting glucose* & 0.061 & 0.477 \\
Creatinine* & 0.020 & 0.832 \\
eGFR & -0.128 & 0.122 \\
total cholesterol & -0.038 & 0.694 \\
Triglyceride* & 0.151 & 0.116 \\
LDL cholesterol* $^{*}$ & -0.028 & 0.779 \\
HDL cholesterol* & -0.073 & 0.459 \\
ACR(mg/g) & -0.028 & 0.781 \\
\hline Dat a & &
\end{tabular}

Data are shown as Pearson correlation coefficients $(r)$. baPWV was log-transformed when statistics were applied. *Denotes the variables that were log-transformed when statistics were applied. 
of hypertension, diabetic retinopathy, diabetic peripheral neuropathy and diabetic nephropathy were 54.7, 16.9, 31.1 and $22.2 \%$, respectively. Prevalence of hypertension was $54.7 \%$ and the information of anti-hypertensives use was shown in Table 1. Furthermore, prevalences of early, definite and severe cardiac autonomic neuropathy were 39.2, 52.7 and $7.4 \%$, respectively.

\section{Correlation between baPWV and other clinical variables}

Table 2 shows the relationships between baPWV and other clinical variables. baPWV was significantly correlated with age $(\mathrm{r}=0.404, \mathrm{p}<0.001)$ and systolic blood pressure $(\mathrm{r}=0.654, \mathrm{p}<0.001)$. Our results indicated that baPWV was not associated with duration of diabetes,
BMI, Waist-to-hip ratio, HbA1c fasting glucose, creatinine, eGFR and lipid variables.

\section{Characteristics of participants according to the presence} of cardiac autonomic neuropathy

Shown in Table 3, subjects with CAN were older and had larger $\operatorname{BMI}(25.0 \pm 3.6$ vs. $23.9 \pm 3.1, \mathrm{p}=0.042)$ and higher baPWV(1722.0(1493.9-2040.8) vs. 1584.0(1446.11812.63), $\mathrm{p}=0.011)$. The prevalence of diabetic peripheral neuropathy was higher $(38.5 \%$ vs $22.9 \%, \mathrm{p}=0.041)$ and NSS score was higher in subjects with CAN. Other microvascular complications including diabetic retinopathy and diabetic peripheral neuropathy did not significantly differ between the two groups. Duration of diabetes, the presence of hypertension and other biochemical

Table 3 Comparison of clinical characteristics according the presence of cardiac autonomic neuropathy

\begin{tabular}{|c|c|c|c|}
\hline Variable & without CAN $(n=70)$ & with CAN $(n=78)$ & $P$ value \\
\hline Age(years) & $57.6 \pm 7.6$ & $61.8 \pm 7.4$ & 0.001 \\
\hline Male,n(\%) & $41(58.6)$ & $39(50.0)$ & 0.296 \\
\hline Current smoker, n (\%) & $17(24.3)$ & $26(33.3)$ & 0.226 \\
\hline Alcohol consumption, n (\%) & 15(21.4) & $22(28.2)$ & 0.342 \\
\hline Hypertension, n (\%) & $35(50.0)$ & $46(59.0)$ & 0.273 \\
\hline Use of $\beta$-Blockers & 16(22.9) & $18(23.1)$ & 0.856 \\
\hline Use of ACEI/ARB & $32(45.7)$ & $35(44.9)$ & 0.746 \\
\hline Use of CCB & 22(31.4) & 26(33.3) & 0.246 \\
\hline Other antihypertensives & $8(11.4)$ & $6(7.7)$ & 0.168 \\
\hline Duration of diabetes(years)* & $6.0(2.0-10.3)$ & $6.0(2.0-11.0)$ & 0.815 \\
\hline $\mathrm{BMI}\left(\mathrm{kg} / \mathrm{m}^{2}\right)$ & $23.9 \pm 3.1$ & $25.0 \pm 3.6$ & 0.042 \\
\hline Waist-to-hip ratio* & $0.94(0.88-0.99)$ & $0.94(0.90-1.00)$ & 0.849 \\
\hline systolic blood pressure(mmHg) & $132.2 \pm 16.4$ & $136.0 \pm 19.4$ & 0.207 \\
\hline diastolic blood pressure $(\mathrm{mmHg})$ & $78.7 \pm 11.0$ & $78.0 \pm 10.3$ & 0.691 \\
\hline $\mathrm{HbA} 1 \mathrm{c}(\%)$ & $7.9 \pm 1.7$ & $8.1 \pm 2.0$ & 0.613 \\
\hline fasting glucose $(\mathrm{mmol} /)^{*}$ & $7.1(6.0-9.1)$ & $6.7(5.8-9.3)$ & 0.750 \\
\hline Creatinine $(\mu \mathrm{mol} / \mathrm{L})^{*}$ & $64.0(52.0-78.0)$ & $59.0(53.0-79.5)$ & 0.519 \\
\hline $\operatorname{eGFR}\left(\mathrm{ml} / \mathrm{min} / 1.73 \mathrm{~m}^{2}\right)$ & $107.8 \pm 25.4$ & $103.0 \pm 31.1$ & 0.303 \\
\hline total cholesterol(mmol/l) & $4.83 \pm 1.92$ & $4.94 \pm 1.16$ & 0.709 \\
\hline Triglyceride $(\mathrm{mmol} / \mathrm{l})^{*}$ & $1.54(0.99-2.69)$ & $1.75(1.10-2.33)$ & 0.510 \\
\hline LDL cholesterol $(\mathrm{mmol} / \mathrm{l})^{*}$ & $2.84(2.11-3.60)$ & $2.83(2.37-3.60)$ & 0.632 \\
\hline $\mathrm{HDL}$ cholesterol $(\mathrm{mmol} / \mathrm{l})^{*}$ & $1.10(0.92-1.30)$ & 1.09(0.94-1.36) & 0.693 \\
\hline $\mathrm{ACR}(\mathrm{mg} / \mathrm{g})$ & 10.90(6.87-24.72) & $15.41(7.60-23.67)$ & 0.472 \\
\hline $\mathrm{baPWV}(\mathrm{cm} / \mathrm{sec})^{*}$ & 1584.0(1446.1-1812.63) & 1722.0(1493.9-2040.8) & 0.011 \\
\hline Diabetic retinopathy, n (\%) & $10(14.3)$ & 15(19.2) & 0.423 \\
\hline Diabetic peripheral neuropathy, n (\%) & 16(22.9) & $30(38.5)$ & 0.041 \\
\hline NSS score* & $3.0(2.0-5.0)$ & $5.0(3.0-6.0)$ & 0.036 \\
\hline NDS score* & $3.0(2.0-4.0)$ & $4.0(2.0-5.0)$ & 0.065 \\
\hline Diabetic nephropathy, n (\%) & $16(22.9)$ & $17(21.8)$ & 0.877 \\
\hline
\end{tabular}

BMI, body mass index; eGFR, modification of diet in renal disease study-glomerular filtration rate. Data are means SD, median (25-75\%), or number (percent).

*Denotes the variables that were log-transformed when statistics were applied. 
findings did not differ significantly according to the presence of CAN.

\section{baPWV and CAN in patients with Type 2 diabetes}

Age greater than 60 years old, BMI and baPWV were significant risk factors for CAN (Model A in Table 4); however, adjusted for age, gender, duration of diabetes, BMI, HbA1c, systolic blood pressure, the presence of diabetic retinopathy, the independent risk factors remained baPWV (odds ratio $=8.496,95 \%$ CI 1.216-59.348; $\mathrm{P}=0.031$ ).

Remarkably, baPWV increased gradually with increasing number of abnormal CARTs (Figure 1). The mean values (SE) of baPWV significantly increased for those with one to above three CARTs were 1647 (44.76), 1663 (36.82), 1911 (91.95) and $2076(127.40) \mathrm{cm} / \mathrm{sec}$ after adjustment for age (per decade), gender, smoking, duration of diabetes. As shown in Table 5, baPWV was significantly higher in patients with abnormal heart rate variation during deep breathing and HR response to standing tests, compared to patients with normal and borderline result tests while there was no such difference observed regarding the valsalva maneuver, postural BP change and sustained handgrip test. After adjustment for age, gender and duration of diabetes, the difference in baPWV between patients with normal and abnormal tests remained significant for heart rate variation during deep breathing $(\mathrm{p}=0.002)$ and $\mathrm{HR}$ response to standing tests $(p=0.046)$.

\section{Discussion}

The present study demonstrates that baPWV was positively associated with the prevalence of cardiac autonomic neuropathy in Type 2 diabetes. These relationships persisted in the multivariate model after adjustment for known risk factors. baPWV was known as a good surrogate markers of clinical atherosclerosis and vascular dysfunction [4]. Our results suggested that CAN might be an important factor of clinical atherosclerosis in T2DM. This finding of the association between CAN and baPWV (a good sign of vascular dysfunction) may help to explain the excess

Table 4 Relationships between multiple risk factors and cardiovascular autonomic neuropathy

\begin{tabular}{|c|c|c|c|c|}
\hline \multirow[t]{2}{*}{ Variable } & \multicolumn{2}{|l|}{ Model A } & \multicolumn{2}{|l|}{ Model B } \\
\hline & Adjusted odds ratio $(95 \% \mathrm{Cl})$ & $\mathbf{P}$ & Adjusted odds ratio $(95 \% \mathrm{Cl})$ & $\mathbf{P}$ \\
\hline Age $>60$ years, no vs yes & $2.247(1.160-4.349)$ & 0.016 & $2.837(1.365-5.897)$ & 0.005 \\
\hline Gender(male) & $1.513(0.776-2.951)$ & 0.225 & $1.692(0.840-3.406)$ & 0.141 \\
\hline Duration of diabetes(per decade) & $0.848(0.474-1.517)$ & 0.578 & $0.832(0.454-1.523)$ & 0.550 \\
\hline $\mathrm{BMI}($ per kg/m2) & $0.888(0.800-0.985)$ & 0.024 & $0.888(0.798-0.988)$ & 0.029 \\
\hline Waist-to-hip ratio* & $2.205(0.014-353.823)$ & 0.760 & $2.119(0.320-14.033)$ & 0.436 \\
\hline systolic blood pressure(per 10 mmHg) & $1.008(0.989-1.027)$ & 0.391 & $1.017(0.996-1.038)$ & 0.108 \\
\hline diastolic blood pressure(per 10 mmHg) & $0.997(0.966-1.028)$ & 0.840 & $0.977(0.919-1.039)$ & 0.457 \\
\hline Current smoker, no vs yes & $1.660(0.792-3.479)$ & 0.179 & $1.331(0.599-2.955)$ & 0.482 \\
\hline Alcohol consumption, no vs yes & $1.521(0.702-3.292)$ & 0.287 & $1.474(0.660-3.293)$ & 0.344 \\
\hline Hypertension, no vs yes & $1.262(0.645-2.468)$ & 0.497 & $1.563(0.767-3.183)$ & 0.219 \\
\hline HbA1c(per 1\%) & $1.115(0.929-1.338)$ & 0.244 & $1.091(0.905-1.314)$ & 0.550 \\
\hline fasting glucose(per $1 \mathrm{mmol} / \mathrm{l})^{*}$ & $1.020(0.922-1.128)$ & 0.700 & $0.594(0.176-2.007)$ & 0.401 \\
\hline Creatinine $(\text { per } 1 \mu \mathrm{mol} / \mathrm{L})^{*}$ & $0.954(0.230-3.950)$ & 0.948 & $1.119(0.256-4.882)$ & 0.881 \\
\hline eGFR( per $1 \mathrm{ml} / \mathrm{min} / 1.73 \mathrm{~m} 2$ ) & $0.999(0.987-1.011)$ & 0.874 & $0.995(0.982-1.008)$ & 0.465 \\
\hline total cholesterol(per $1 \mathrm{mmol} / \mathrm{l})$ & $1.046(0.807-1.355)$ & 0.735 & $1.024(0.778-1.349)$ & 0.864 \\
\hline Triglyceride(per $1 \mathrm{mmol} / \mathrm{l})^{*}$ & $0.823(0.462-1.469)$ & 0.511 & $0.969(0.524-1.795)$ & 0.921 \\
\hline LDL cholesterol(per $1 \mathrm{mmol} / \mathrm{l})^{*}$ & $1.405(0.426-4.634)$ & 0.576 & $1.470(0.423-5.113)$ & 0.545 \\
\hline $\mathrm{HDL}$ cholesterol(per $1 \mathrm{mmol} / \mathrm{l})^{*}$ & $0.740(0.242-2.267)$ & 0.598 & $0.632(0.196-2.034)$ & 0.442 \\
\hline $\mathrm{ACR}(\mathrm{mg} / \mathrm{g})$ & $1.000(0.999-1.000)$ & 0.574 & $1.000(0.999-1.000)$ & 0.538 \\
\hline $\mathrm{baPWV}(\mathrm{cm} / \mathrm{sec})^{*}$ & $6.117(1.032-36.245)$ & 0.046 & $8.496(1.216-59.348)$ & 0.031 \\
\hline Diabetic retinopathy, no vs yes & $1.431(0.586-3.493)$ & 0.431 & $1.589(0.634-3.984)$ & 0.323 \\
\hline Diabetic peripheral neuropathy, no vs yes & $2.071(0.994-4.315)$ & 0.052 & $2.672(1.155-6.183)$ & 0.022 \\
\hline Diabetic nephropathy, no vs yes & $0.940(0.426-2.072)$ & 0.878 & $1.087(0.459-2.575)$ & 0.849 \\
\hline
\end{tabular}

Data were obtained from a multivariate regression model.

Model A, adjusted for age; Model B, adjusted for age, gender, smoking, drinking, duration of diabetes, BMI, HbA1c, systolic blood pressure, the presence of diabetic retinopathy.

*Denotes the variables that were log-transformed when statistics were applied. 


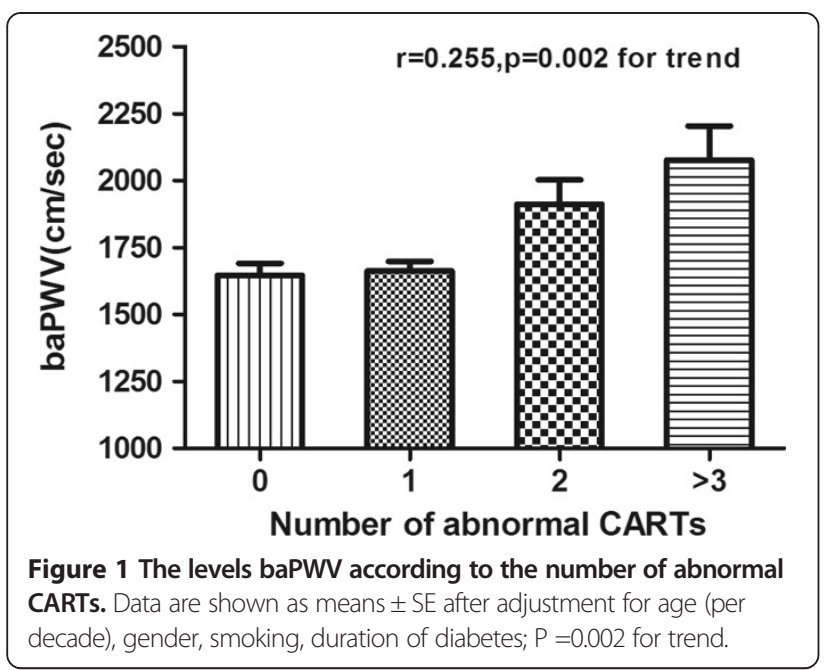

cardiovascular disease mortality seen in those with T2DM subjects with CAN.

The main pathological findings for CAN are damages to the autonomic nerve fibers that innervate the heart and blood vessels and this resulted in abnormalities in heart rate control and vascular dynamics [3]. Previous studies have reported that CAN was associated with accelerated atherosclerosis, represented as carotid intima media thickness (CIMT) and plaques, independent of the traditional cardiovascular risk factors in T2DM $[15,16]$. Therefore, the presence of CAN should be considered and accessed when patients are in the early stage of diabetes, rather than after the development of clinical cardiovascular disease.

Pulse wave velocity describes the rigidity of the arterial wall, and is an indicator of atherosclerosis irrespective of classical cardiovascular risk factors and ethnicity [17]. Many prospective studies showed that PWV had more advantages in reflecting the risk of cardiovascular disease than the blood pressure and had a strong relationship with cardiovascular events and all-cause mortality [18]. As a simpler noninvasive method, brachial-ankle PWV (baPWV) integrates the mechanical properties from both the central and peripheral arteries. It has a high correlation with traditional carotid-femoral PWV (cfPWV), and it is suitable for general population studies [6]. Previous studies have indicated that baPWV was not only directly related to macrovascular complications but also microangiopathic conditions and diabetes peripheral neuropathy. Yokoyama et al. reported that baPWV, retinopathy, age, and glycated hemoglobin were independent risk factors in DPN and the associated autonomic neuropathy [19]. Byung et al. reported that increased baPWV was significantly correlated with peripheral neuropathy in patients with type 2 diabetes which was diagnosed by total symptom score (TSS) and abnormal neurological assessment [20]. As to the relationship between baPWV and CAN, a recent study [8] pointed out that the association between PWV and CAN was independently significant in diabetes, which used heart rate variability (HRV) as an index for CAN. Our study further confirmed the association between CAN and baPWV using CARTs as diagnosis criterion for CAN. In a univariate analysis of the risk factors for CAN, the odds ratio for baPWV was 6.117(95\% CI, 1.032-36.245; $P=0.046)$, which is a meaningful risk factor for CAN. A multivariate logistic regression analysis was performed to adjust for confounding variables and the adjusted odds ratio was $8.496(95 \% \mathrm{CI}, 1.216-59.348 ; \mathrm{P}=0.031)$, which indicates that baPWV might be an independent risk factor for CAN.

Previous researches pointed out the primary factors affecting baPWV are age, systolic blood pressure, and gender [21]. In a Korean study on type 2 diabetes patients, baPWV was significantly correlated with mean blood pressure, heart rate, age, urine albumin/creatinine ratio [22]. In our study, age, systolic blood pressure and diastolic blood pressure were positive related with baPWV. Duration of diabetes, BMI, glycated hemoglobin, lipid levels were not correlated with baPWV. To our surprise, urine albumin/creatinine ratio which have been proved to be significantly associated with arteriosclerosis and autonomic function $[23,24]$ was not related with baPWV in our study. That may be because $85.0 \%$ of our patients had normal urine albumin/creatinine ratio which weaken the link between urine albumin/creatinine ratio and baPWV.

According to our results, baPWV was significantly higher in patients with abnormal heart rate variation during deep breathing and HR response to standing tests. Both heart rate response to deep breathing (deep breathing test) and heart rate response to standing (standing test)

Table 5 Mean baPWV value in patients with normal, borderline, and abnormal autonomic nervous function tests

\begin{tabular}{llllll}
\hline & & Normal result & Borderline result & Abnormal result & $P$ for trend \\
\hline baPWV(cm/sec)* & HR variation during deep breathing & $1537.50(1392.88-1863.00)$ & $1649.25(1480.75-1815.50)$ & $1728.25(1524.00-2029.38) \# \#$ & 0.002 \\
& HR response to standing & $1623.75(1444.38-1890.00)$ & $1757.25(1552.75-2012.75)$ & $1825.00(1509.88-2020.63) \#$ & 0.046 \\
& Valsalva maneuver & $1666.25(1459.88-1945.75)$ & $1642.00(1512.25-2006.50)$ & $1669.50(1445.25-1934.75)$ & 0.392 \\
& postural BP change & $1651.75(1486.88-1937.00)$ & $1661.25(1426.50-1983.50)$ & $1775.86(1568.67-2043.00)$ & 0.471 \\
& Sustained handgrip test & $1656.00(1500.13-1947.50)$ & $1540.75(1329.37-1939.63)$ & $1777.25(1509.88-1943.38)$ & 0.835 \\
\hline
\end{tabular}

$\mathrm{HR}$, heart rate; $\mathrm{BP}$, blood pressure.

*Denotes the variables that were log-transformed when statistics were applied.

$\# \mathrm{P}<0.05$, \#\#P $<0.01$, for comparisons of baPWV values between abnormal, borderline and normal test. 
which concerns heart rate variation), reflect mainly parasympathetic activity of the heart innervation although the sympathetic nervous system may somewhat affect these reactions $[3,25]$. But heart rate variation during the Valsalva maneuver and sustained handgrip test is mediated by combined effect of parasympathetic and sympathetic nerve fibres $[3,26]$. Our study showed that the increase of baPWV better reflected the damage of parasympathetic nerve fibres and for those CARTs who have a dual innervation (parasympathetic and sympathetic nervous system), the change of baPWV was insensitive. Our study also demonstrated that the number of abnormal CARTs increased gradually with increasing baPWV. In other word, the surge of baPWV is a comprehensive reflection of injuries of the sympathetic nervous system and the parasympathetic nervous system. The association between PWV and cardiac autonomic function also has been examined in a small study in healthy individuals [27]. Consistent with our results, heart rate response to deep breathing showed moderate correlations with PWV. CARTs mainly characterizing sympathetic function had no correlation with aortic stiffness parameters including PWV.

The relationship between the decrease of large arteries elasticity and the dysfunction of autonomic nervous system in type 2 diabetes is not fully understood. It's not clear whether arterial stiffness produce dysfunction of the cardiac autonomic nervous system or CAN induce stiffness of the large arteries. Some study suggested that dysfunction of the cardiovascular autonomic system may influence the elasticity of the arterial wall by affecting the vascular tone of large arteries [28]. Other studies indicated that tachycardia which induced by CAN is main reason for arterial stiffness. Indeed, increases in heart rate lead to arterial stiffness independently of changes in activity of the autonomic nervous system [29]. The present analysis showed that there was correlation between heart rate response to deep breathing, standing and baPWV, which partly showed the association between parasympathetic dysfunction and arterial stiffness.

The limitations of our study resulted from retrospective cross-sectional design, which did not allow drawing conclusions about any causal relation between CAN and baPWV. We also could not assess how the changes in both CARTs and baPWV through time, as well as the changes in the serum glucose levels and glycosated hemoglobin, affect each other. Secondly, our study was conducted in outpatient department of endocrinology and we formulated strict exclusion criteria. It largely decreased the number of patients in our study. CART tests required patients to actively cooperate with us and the entire check need relatively long time. Many elderly people and patients with severe diabetic complication refused to participate in our study or dropped out from the study. So the participants in our study were younger and had better health status, which means the conclusion of our study may not be applied to other diabetes patients. Moreover, the effects of the type of diabetes treatment and other prescribed medications on CAN and PWV need to be investigated in future studies.

In conclusion, CAN, particularly the injury of parasympathetic activity of the heart, as expressed by abnormal heart rate variation during deep breathing and standing, correlates strongly with baPWV in patients with type 2 diabetes. Most interestingly, this finding was observed in a population without apparent history of cardiovascular condition.

\section{Abbreviation}

CAN: Cardiac autonomic neuropathy; baPWV: Brachial-ankle pulse wave velocity; CARTs: Cardiovascular reflex tests; BP: Blood pressure; T2DM: Type 2 diabetes mellitus; HRV: Heart rate variability; HR: Heart rate; DR: Diabetic retinopathy; DPN: Diabetic peripheral neuropathy; BMI: Body mass index; eGFR: Modification of diet in renal disease study-glomerular filtration rate; ACR: Albumin-to-creatinine ratio.

\section{Competing interests}

The authors declare that they have no competing interests.

\section{Authors' contribution}

NW drafted the manuscript. All authors participated in the conception and design of the study. XC, KY, YL and MH performed the statistical analysis. NW and $\mathrm{KY}$ performed cardiac autonomic function test and measured baPWV. YL and $\mathrm{MH}$ collected patients' information. All authors read and approved the final manuscript.

\section{Acknowledgements}

This project was supported by the National Natural Science Foundation of China (81270902, 81030014, 81120004)

\section{Author details}

${ }^{1}$ The Institute of Endocrinology and Diabetology, Huashan Hospital, Shanghai Medical College, Fudan University, 12 Middle Wulumuqi Road, Shanghai, China. ${ }^{2}$ The Emergency Department, Zhongshan Hospital, Shanghai Medical College, Fudan University, 180 Fenglin Road, Shanghai, China.

Received: 29 April 2014 Accepted: 10 July 2014

Published: 30 July 2014

\section{References}

1. Maser RE, Mitchell BD, Vinik Al, Freeman R: The association between cardiovascular autonomic neuropathy and mortality in individuals with diabetes: a meta-analysis. Diabetes Care 2003, 26(6):1895-1901.

2. Kuehl M, Stevens MJ: Cardiovascular autonomic neuropathies as complications of diabetes mellitus. Nat Rev Endocrinol 2012, 8(7):405-416.

3. Vinik Al, Ziegler D: Diabetic cardiovascular autonomic neuropathy. Circulation 2007, 115(3):387-397.

4. van Popele NM, Grobbee DE, Bots ML, Asmar R, Topouchian J, Reneman RS, Hoeks AP, van der Kuip DA, Hofman A, Witteman JC: Association between arterial stiffness and atherosclerosis: the Rotterdam Study. Stroke 2001, 32(2):454-460

5. Aso K, Miyata M, Kubo T, Hashiguchi H, Fukudome M, Fukushige E, Koriyama N, Nakazaki M, Minagoe S, Tei C: Brachial-ankle pulse wave velocity is useful for evaluation of complications in type 2 diabetic patients. Hypertens Res 2003, 26(10):807-813.

6. Yu WC, Chuang SY, Lin YP, Chen CH: Brachial-ankle vs carotid-femoral pulse wave velocity as a determinant of cardiovascular structure and function. J Hum Hypertens 2008, 22(1):24-31.

7. European Society of Hypertension-European Society of Cardiology Guidelines Committee: 2003 European society of hypertension-European society of 
cardiology guidelines for the management of arterial hypertension. J Hypertens 2003, 21(6):1011-1053.

8. Bagherzadeh A, Nejati-Afkham A, Tajallizade-Khoob Y, Shafiee A, Sharifi F, Esfahani MA, Badamchizade Z, Alatab S, Fakhrzadeh H: Association of cardiac autonomic neuropathy with arterial stiffness in type 2 diabetes mellitus patients. J Diab Metab Disord 2013, 12(1):55.

9. Ewing DJ, Martyn CN, Young RJ, Clarke BF: The value of cardiovascular autonomic function tests: 10 years experience in diabetes. Diabetes Care 1985, 8(5):491-498

10. O'Brien IA, O'Hare JP, Lewin IG, Corrall RJ: The prevalence of autonomic neuropathy in insulin-dependent diabetes mellitus: a controlled study based on heart rate variability. Q J Med 1986, 61(234):957-967.

11. Ohnishi H, Saitoh S, Takagi S, Ohata J, Isobe T, Kikuchi Y, Takeuchi H, Shimamoto K: Pulse wave velocity as an indicator of atherosclerosis in impaired fasting glucose: the Tanno and Sobetsu study. Diabetes Care 2003, 26(2):437-440.

12. Scanlon PH, Foy C, Malhotra R, Aldington SJ: The influence of age, duration of diabetes, cataract, and pupil size on image quality in digital photographic retinal screening. Diabetes Care 2005, 28(10):2448-2453.

13. KDOQI work group: KDOQI clinical practice guidelines and clinical practice recommendations for diabetes and chronic kidney disease. Am J Kidney Dis 2007, 49(2 Suppl 2):S12-S154.

14. Tesfaye S, Boulton AJ, Dyck PJ, Freeman R, Horowitz M, Kempler P, Lauria G, Malik RA, Spallone V, Vinik A, Bernardi L, Valensi P, Toronto Diabetic Neuropathy Expert Group: Diabetic neuropathies: update on definitions, diagnostic criteria, estimation of severity, and treatments. Diabetes Care 2010, 33(10):2285-2293.

15. Jung $\mathrm{CH}$, Baek AR, Kim KJ, Kim BY, Kim CH, Kang SK, Mok JO: Association between cardiac autonomic neuropathy, diabetic retinopathy and carotid atherosclerosis in patients with type 2 diabetes. Endocrinol Metab (Seoul) 2013, 28(4):309-319.

16. Sinha PK, Santr G, De D, Sah A, Biswas K, Bhattachary P, Ghosh P: Carotid intima-media thickness in type 2 diabetes mellitus patients with cardiac autonomic neuropathy. J Assoc Physicians India 2012, 60:14-18

17. Krantz MJ, Long CS, Hosokawa P, Karimkahani E, Dickinson M, Estacio RO, Masoudi FA, Havranek EP: Pulse wave velocity and carotid atherosclerosis in white and Latino patients with hypertension. BMC Cardiovasc Disord 2011, 11:15.

18. Vlachopoulos C, Aznaouridis K, Stefanadis C: Prediction of cardiovascular events and all-cause mortality with arterial stiffness: a systematic review and meta-analysis. J Am Coll Cardiol 2010, 55(13):1318-1327.

19. Yokoyama H, Shoji T, Kimoto E, Shinohara K, Tanaka S, Koyama H, Emoto M, Nishizawa Y: Pulse wave velocity in lower-limb arteries among diabetic patients with peripheral arterial disease. J Atheroscler Thromb 2003, 10(4):253-258

20. Ha BK, Kim BG, Kim DH, Lee SI, Jung SM, Park JY, Lee CW, Kim SS, Kim BH, Kim IJ: Relationships between Brachial-Ankle Pulse Wave Velocity and Peripheral Neuropathy in Type 2 Diabetes. Diab Metab J 2012, 36(6):443-451.

21. Kubo T, Miyata M, Minagoe S, Setoyama S, Maruyama I, Tei C: A simple oscillometric technique for determining new indices of arterial distensibility. Hypertens Res 2002, 25(3):351-358.

22. Choi BG, Kang JH, Jeon YK, Kim SS, Lee CW, Kim IJ, Kim YK, Kim BH: Determinants of brachial-ankle pulse wave velocity in normotensive young adults with type 2 diabetes mellitus. J Korean Med Sci 2012, 27(11):1359-1363.

23. Meisinger $C$, Heier M, Landgraf R, Happich M, Wichmann HE, Piehlmeier W: Albuminuria, cardiovascular risk factors and disease management in subjects with type 2 diabetes: a cross sectional study. BMC Health Serv Res 2008, 8:226.

24. Jaiswal M, Urbina EM, Wadwa RP, Talton JW, D'Agostino RJ, Hamman RF, Fingerlin TE, Daniels S, Marcovina SM, Dolan LM, Dabelea D: Reduced heart rate variability among youth with type 1 diabetes: the SEARCH CVD study. Diabetes Care 2013, 36(1):157-162.

25. Pfeifer MA, Cook D, Brodsky J, Tice D, Reenan A, Swedine S, Halter JB, Porte DJ: Quantitative evaluation of cardiac parasympathetic activity in normal and diabetic man. Diabetes 1982, 31(4 Pt 1):339-345.

26. Vinik Al, Maser RE, Mitchell BD, Freeman R: Diabetic autonomic neuropathy. Diabetes Care 2003, 26(5):1553-1579.

27. Nemes A, Takacs R, Gavaller H, Varkonyi TT, Wittmann T, Forster T, Lengyel C: Correlations between aortic stiffness and parasympathetic autonomic function in healthy volunteers. Can J Physiol Pharmacol 2010, 88(12):1166-1171.
28. Frattola A, Parati G, Gamba P, Paleari F, Mauri G, Di Rienzo M, Castiglioni P, Mancia G: Time and frequency domain estimates of spontaneous baroreflex sensitivity provide early detection of autonomic dysfunction in diabetes mellitus. Diabetologia 1997, 40(12):1470-1475.

29. Mircoli L, Mangoni AA, Giannattasio C, Mancia G, Ferrari AU: Heart ratedependent stiffening of large arteries in intact and sympathectomized rats. Hypertension 1999, 34(4 Pt 1):598-602.

doi:10.1186/1758-5996-6-82

Cite this article as: Wu et al: Association between Brachial-Ankle pulse wave velocity and cardiac autonomic neuropathy in type 2 diabetes. Diabetology \& Metabolic Syndrome 2014 6:82.

\section{Submit your next manuscript to BioMed Central and take full advantage of:}

- Convenient online submission

- Thorough peer review

- No space constraints or color figure charges

- Immediate publication on acceptance

- Inclusion in PubMed, CAS, Scopus and Google Scholar

- Research which is freely available for redistribution

Submit your manuscript at www.biomedcentral.com/submit
Ciomed Central 